\title{
How Factors Involved in the Resolution of Crystal-Induced Inflammation Target IL-1 $\beta$
}

\author{
Francesca Oliviero* and Anna Scanu \\ Rheumatology Unit, Department of Medicine - DIMED, University of Padova, Padova, Italy
}

One of the main clinical features characterizing crystal-induced inflammation is its spontaneous resolution. The aim of this review is to outline the various factors involved in the self-limiting course of crystal-induced inflammation focusing on their effect on $\mathrm{IL}-1 \beta$ production. Endogenous molecules that are induced or locally recruited by the process itself, inhibitory proteins naturally present in the joint and exogenous dietary factors are discussed. Aside from the classical well-known molecules involved in the resolution of crystal-induced acute attack such as TGF $\beta$, IL-10, IL-1Ra, and lipoproteins,

OPEN ACCESS

Edited by:

Annalisa Bruno,

University of Chieti-Pescara, Italy

Reviewed by:

Paola Bossù,

Fondazione Santa Lucia (IRCCS), Italy

Flavio Almeida Amaral,

Universidade Federal de Minas

Gerais, Brazil

Elena Ossipova,

Karolinska Institutet, Sweden

*Correspondence:

Francesca Oliviero

francesca.oliviero@unipd.it

Specialty section:

This article was submitted to Inflammation Pharmacology,

a section of the journal

Frontiers in Pharmacology

Received: 10 January 2017 Accepted: 13 March 2017

Published: 28 March 2017

Citation:

Oliviero F and Scanu A (2017) How Factors Involved in the Resolution of Crystal-Induced Inflammation

Target IL-1 $\beta$.

Front. Pharmacol. 8:164. doi: 10.3389/fphar.2017.00164 particular attention is paid to recently uncovered mechanisms such as the aggregation of neutrophil extracellular traps, the release of ectosomes from neutrophil surface, and alpha-1-anti-trypsin-mediated IL-1 inhibition.

Keywords: crystal-induced inflammation, interleukin-1, transforming growth factor, lipoproteins, neutrophil extracellular traps, dietary factors

\section{INTRODUCTION}

Crystal-induced inflammation is caused by the presence of monosodium urate (MSU) or calcium pyrophosphate (CPP) crystals in articular or periarticular tissues. Although MSU and CPP crystals form in different ways, their effects are very similar and associated with an acute, intense inflammatory reaction characterized by massive leukocyte recruitment and the local release of cytokines, chemokines, reactive oxygen species and proteolytic enzymes (Liu-Bryan and Lioté, 2005).

Since the time cytoplasmic NACHT-LRRPYD-containing protein-3 (NLRP) 3 inflammasome was first identified and its activation by MSU and CPP crystals was demonstrated (Martinon et al., 2006), interleukin (IL)-1 $\beta$ has been considered the most important inflammatory mediator in crystal-induced inflammation, and it represents one of the main targets for new drugs that have been or are being developed to treat gout and calcium crystal-induced arthritis (Dinarello, 2014).

Although the molecular mechanisms leading to the activation of NLRP3 by pathogenic crystals have not been fully elucidated, the two-step process linked to the production of IL- $1 \beta$ has been clearly demonstrated. The first signal is triggered by pattern-recognition receptors (e.g., TLRs) which initiate the transcription of IL- $1 \beta$; the second signal triggers inflammasome activation, which in turn activates caspase- 1 and leads to the cleavage of the IL- $1 \beta$ precursors into the active cytokine (Joosten et al., 2010; Netea et al., 2015; Scanu et al., 2016). Once released, the cytokine promotes the induction of different pro-inflammatory genes amplifying the inflammatory process and leading to long term articular damage. Similarly to IL-1 $\beta$, IL-18 is produced by caspase- 1 activation but its role in crystal-induced inflammation has not been clearly defined. 
One of the main clinical features characterizing crystalinduced inflammation is its spontaneous resolution. It is wellknown that patients experiencing an acute attack improve within a few days time and that the disease progresses to a chronic state only if untreated (Punzi et al., 2012). The various factors involved in the self-limiting course of crystal-induced inflammation are outlined in this review. Most of these are endogenous molecules that are induced or locally recruited by the process itself or are inhibitory proteins naturally present in the joint. Other factors are exogenous dietary substances that can modulate the resolution of the acute attack (Figure 1).

An in-depth analysis of these mechanisms reveals that the final outcome is a direct or indirect action on IL-1 $\beta$ production. In particular, a negative regulation of inflammasome activation and pro-IL-1 $\beta$ expression have been described.

\section{Transforming Growth Factor}

Transforming growth factor (TGF) $\beta 1$ is one of the main molecules involved in the resolution of crystal-induced inflammation. The effect of TGF $\beta 1$ was initially described in a subcutaneous air pouch animal model in which it inhibited MSU crystal-induced leukocyte chemotaxis (Lioté et al., 1996).

Some investigators hypothesized that the shift from a proinflammatory state to an active production of anti-inflammatory molecules is the mechanism leading to the resolution of an acute attack (Chen et al., 2011). It has been shown, in fact, that the differentiation of monocytes into macrophages and the uptake of crystals by the latter induces TGF $\beta 1$ secretion (Chen et al., 2011). The presence of high levels of TGF $\beta 1$, IL-1 receptor antagonist (Ra), IL-10 and soluble receptors of tumor necrosis factor (TNF) has, in fact, been demonstrated in the synovial fluid (SF) of patients with gout and has been associated with the upregulation of intracellular negative cytokine regulators such as the suppressors of cytokine signalling (SOCS) 3 and the cytokine inducible SH2-containing protein (CIS). While enhancing TGF $\beta 1$ expression, these proteins downregulate IL-1 $\beta$ and TNF secretion (Chen et al., 2011).

In addition, the inverse relationship between TGF $\beta 1$ and IL$1 \beta$ was clearly demonstrated by a study examining different inflammatory mediators in the SF collected during different stages of acute gout attack (Scanu et al., 2012).

A tight regulation of IL- $1 \beta$ production by TGF $\beta 1$ has also been demonstrated with regard to neutrophils. Activated neutrophils have, in fact, been identified as an additional potential source of local TGF $\beta 1$ production. An increased TGF $\beta 1$ expression following neutrophil phagocytosis of apoptotic cells, a process which in turn regulates IL- $1 \beta$ production by the neutrophils themselves, has been described (Steiger and Harper, 2013).

The negative regulation of IL- $1 \beta$ production by the crystalinduced TGF $\beta 1$ signaling pathway has been confirmed by other investigators. In particular, a higher transglutaminase (TG) 2 expression in crystal-exposed macrophages has been linked to TGF $\beta$ production (Yen et al., 2015) which, in turn, downregulates IL-1 $\beta$ release through the inhibition of the Janus kinase (JAK) 2 signaling. TG is indeed highly expressed in gout and has been shown to enhance phagocytosis of apoptotic leukocytes by macrophages limiting neutrophil accumulation and promoting resolution (Rose et al., 2006).

Importantly, TGF $\beta 1$ has been shown to inhibit cell surface IL-1 receptor expression (Dubois et al., 1990) supporting an additional role in limiting crystal-induced inflammatory response.

\section{Lipoproteins}

Several studies have demonstrated the importance of lipoproteins as modulators of crystal-induced inflammation. It has been hypothesized that changes in SF lipoprotein concentration and in the proteins that coat crystals play an integral role in the selflimiting nature of an acute attack. In this context, lipoproteins containing apolipoproteins (apo) B and E, have demonstrated potential regulatory effects suppressing MSU crystal phagocytosis and alpha-mannosidase release by neutrophils (Terkeltaub et al., 1986, 1991). This is probably due to the ability of these proteins to displace complement-activating IgG that initially cover the crystals (Ortiz-Bravo et al., 1993). It has also been hypothesized that the binding of low density (LDL) and high density lipoproteins (HDL) to the crystal surface reduces MSU- and $\mathrm{CPP}$-induced lactate dehydrogenase release from neutrophils, a marker for cytolysis (Burt et al., 1989). It has been shown that the levels of LDL in the rat air-pouch model surge and remain elevated $24 \mathrm{~h}$ after the injection of CPP crystals, while the number of white blood cells and the concentration of $\beta$-Glucoronidase and PGE2 significantly fell (Kumagai et al., 2001). In addition, it has been reported that HDL inhibit MSU crystal-induced CCL2 production and expression in human synoviocytes and reduce monocyte/macrophage recruitment (Scanu et al., 2010).

Although several studies have demonstrated that lipoproteins may contribute to limiting crystal induced inflammation, their effects on the production of IL- $1 \beta$ have been evaluated only for HDL. Air pouch experiments have shown that HDL display antiinflammatory activity reducing the recruitment of leukocytes and affecting pro- and anti-inflammatory cytokine balance after MSU crystal injection. It has been reported, in particular, that HDL reduce the release of IL- $1 \beta$ in pouch exudates and the mRNA levels in membranes. Interestingly, HDL do not affect the production of the crystal-induced anti-inflammatory factor IL-1Ra. Unlike other lipoproteins, HDL may act not only by adhering to the surface of the crystals, but also through a direct interaction with the inflammatory cells (Scanu et al., 2015).

\section{IL-1Ra}

Although IL-1Ra is the natural IL-1 inhibitor as it functions as an IL-1 receptor competitor (Dayer, 2002) and its effectiveness in the treatment of gout has been established, few studies have been conducted to assess its role in the spontaneous resolution of crystal-induced inflammation. Anti-inflammatory cytokine assessment in SF demonstrated that IL-1Ra levels are higher in gouty patients than in osteoarthritis patients (Chen et al., 2011). The injection of MSU crystals into a murine air pouch causes IL-1Ra production after $3 \mathrm{~h}$, although the levels detected are not sufficient to inhibit the inflammatory effect of IL-1 $\beta$ at that same time point (Scanu et al., 2015). In vitro stimulation of monocytes/macrophages by synthetic MSU, CPP and basic 


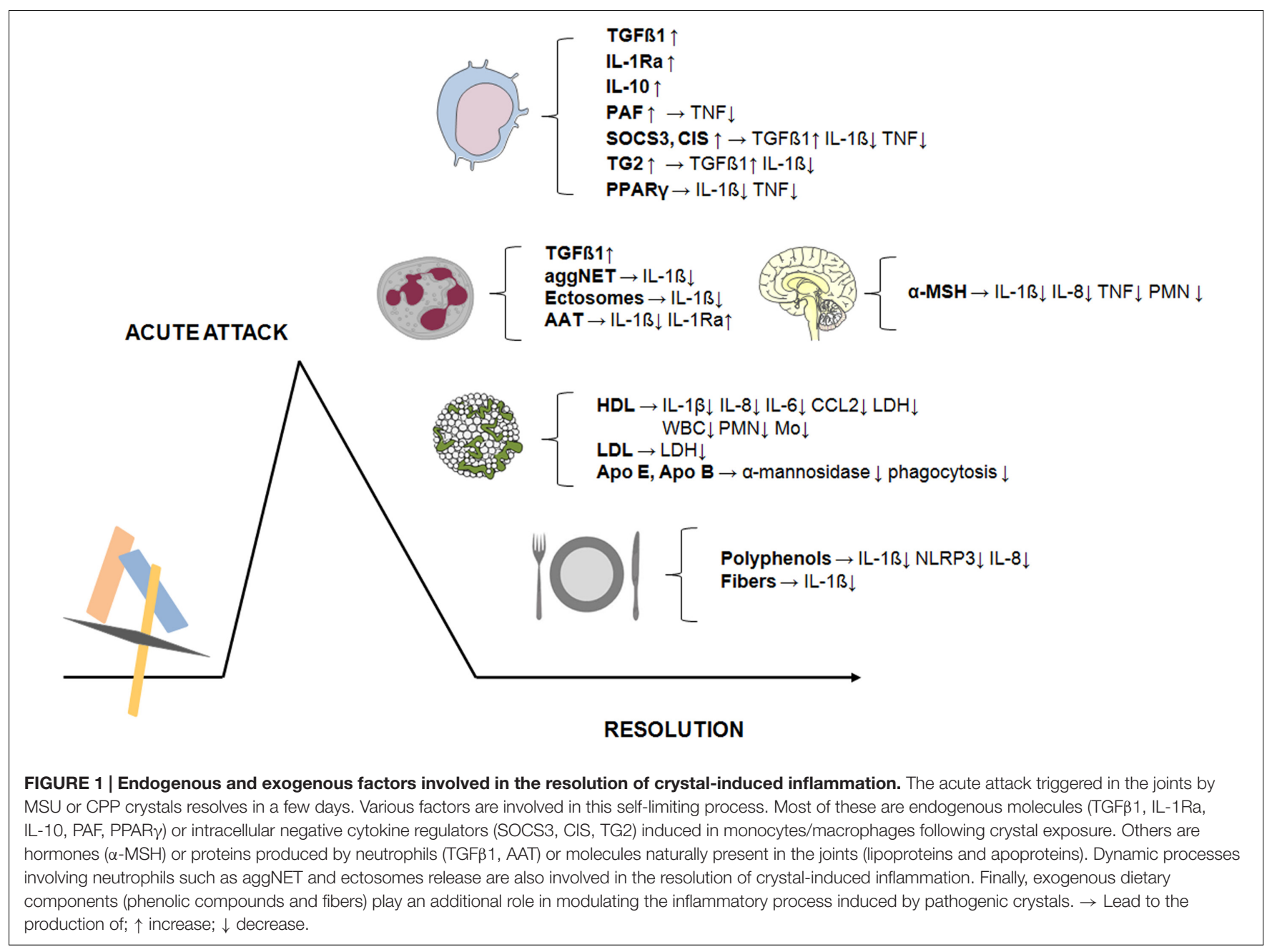

calcium phosphate (BCP) crystals induces a rapid increase in proinflammatory cytokines such as IL- $1 \beta$, IL- 8 and IL-6, whereas longer periods are required to release high levels of IL-1Ra (a personal observation).

\section{Neutrophil Extracellular Traps and Microvesicles}

The aggregation of neutrophil extracellular traps (NETs) induced by pathogenic crystals has been recently associated with the resolution of neutrophilic inflammation that characterizes the acute crystal-induced inflammatory process.

NET formation (NETosis) is accompanied by the release of a variety of pro-inflammatory mediators that orchestrate the local innate immune response. When neutrophils are under conditions of high cell density, crystal-induced NETs form dense aggregates that sequester and degrade neutrophil inflammatory mediators, in particular IL-1 $\beta$. It has been demonstrated that this process is mediated by ROS which, in this particular case, downregulates inflammation (Schauer et al., 2014).

Another phenomenon that is associated with the resolution of crystal-induced arthritis is the release of phosphatidylserine positive ectosomes from the surface of neutrophils during the inflammatory process. It has been demonstrated that these ectosomes suppress inflammasome and consequently inhibit IL- $1 \beta$ release in C5a primed macrophages (Cumpelik et al., 2016). Although ectosomes trigger the release of TGF by monocyte and macrophages, it was not found that TGF was necessary to suppress inflammasome activation.

\section{Other Endogenous Factors}

A variety of other regulatory factors involved in the spontaneous resolution of an acute attack of crystal-induced arthritis have been identified. Among them, peroxisome proliferator-activated receptor $\gamma(\operatorname{PPAR} \gamma)$, a nuclear hormone receptor, has been shown to be expressed in human monocytes after MSU crystal stimulation. PPAR $\gamma$ ligands reduce the crystal-induced production of IL-1 $\beta$ and TNF in vitro (Akahoshi et al., 2003).

Similarly, melacortin receptor (MC-R) agonists can also influence the resolution of an acute gout attack. Selective ligands for MC-R3, such as $\alpha$-melacortin-stimulating hormone ( $\alpha$-MSH), lower the levels of IL-1 $\beta$ and the chemokine (C-X-C motif) ligand 1 (CXCL1) and the polymorphonuclear cell (PMN) migration in a murine model of MSU crystal-induced peritonitis (Getting et al., 2001, 2006). In addition, it was found that $\alpha-\mathrm{MSH}$ inhibits, 


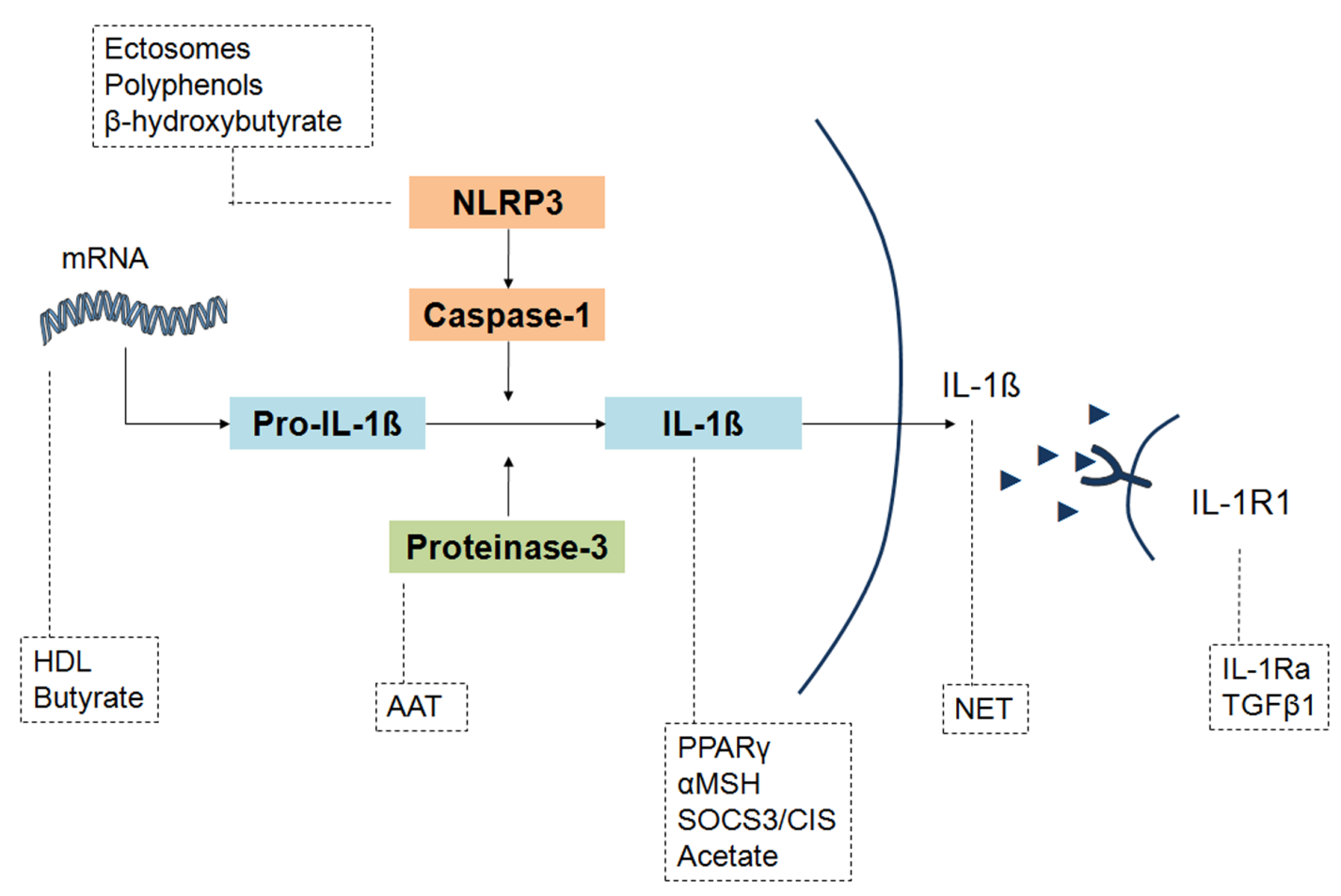

FIGURE 2 | Different levels of IL-1 inhibition by the endogenous and exogenous factors involved in the resolution of crystal-induced inflammation. IL-1 $\beta$ production pathway can be affected by molecules that act upstream on NLRP3 activation (ectosomes, polyphenols, $\beta$-idroxybutyrate) and IL-1 $\beta$ mRNA expression (HDL, butyrate), or downstream on pro-IL-1 $\beta$ cleavage (AAT), IL-1 $\beta$ formation (PPAR $\gamma, \alpha M S H, S O C S 3 / C I S$, acetate) and concentrations (NET), or inhibiting IL-1 $\beta$ bond to its receptor IL-1R1 (IL-1Ra, TGF $\beta 1$ ). Although the final effect of PPAR $\gamma, \alpha M S H$ and acetate is an inhibition of IL-1 $\beta$ levels, their precise mechanism of action has not been elucidated.

in vitro, the capacity of monocytes to release IL-1 $\beta$ and other pro-inflammatory cytokines in response to MSU crystals, without affecting the secretion of caspase-1, the enzyme responsible for converting pro-IL-1 $\beta$ to cytokine's active form (Capsoni et al., 2009). By contrast, $\beta$-hydroxybutyrate (BHB), a ketone body produced in the liver, has been observed to inhibit IL-1 $\beta$ processing in response to MSU crystals by reducing caspase-1 activation. BHB has been shown to block NLRP3 inflammasome preventing the decline of $\mathrm{K}^{+}$intracellular efflux induced by NLRP3 activators (Youm et al., 2015).

Other molecules that may trigger resolution are induced during inflammatory processes promoted by crystals, even though they may play a minor role. Higher levels of IL-10 were observed in the SF from patients with acute gout with respect to OA patients (Chen et al., 2011). But although it has been demonstrated that IL-10 overexpression blocks MSU crystalinduced inflammation, including the suppression of TNF release in vitro and CXCL1 production in vivo (Murakami et al., 2002), there is no evidence of IL-10 in supernatants from differentiated macrophages incubated with MSU crystals (Yagnik et al., 2004).

Macrophage derived platelet activating factor (PAF) and related molecules could, according to one hypothesis, play a role in suppressing the inflammatory response. In vitro experiments demonstrated that MSU crystal-stimulated macrophages release PAF, which in turn downregulates the TNF secretion (Yagnik, 2014).
The anti-inflammatory effect of IL-10 and PAF has not been assessed in the crystal-induced IL-1 $\beta$ production or action/pathway.

A recently described mechanism involved in the inhibition of crystal-induced inflammation concerns alpha-1-anti-trypsin (AAT), the major, natural inhibitor of serine proteases produced by neutrophils that can be found in human serum during infections and inflammation. Serine proteases have been found to be responsible for the caspase-1-independent conversion of IL- $1 \beta$ precursor into the active cytokine in neutrophils (Joosten et al., 2009) which are the main inflammatory cells in crystal arthritis. It has been demonstrated that AAT not only reduces the release and the extracellular processing of IL- $1 \beta$ but also increases circulating levels of endogenous IL-1Ra, the natural inhibitor of IL-1 (Joosten et al., 2016).

\section{Exogenous Factors}

Although, it is well-established that nutrients or dietary metabolites possess immune-regulatory properties and can modulate the inflammatory response (Camell et al., 2015), their role in crystal-induced inflammation has only recently been taken into consideration.

Well-known for their antioxidant, anti-inflammatory and anti-cancer effects, plant polyphenols are the most studied dietary compounds. In particular, green tea epigallocatechin-3gallate (EGCG) has been observed to reduce the inflammatory 
response to CPP crystals by inhibiting IL-1 $\beta$, IL-8, and CCL2 in vitro (Oliviero et al., 2013). An inhibitory effect exerted by EGCG was also demonstrated in urate crystalinduced peritoneal inflammation. A reduction in IL-1 $\beta$ levels in the peritoneal lavage fluid has been observed together with a lower NLRP3 inflammasome expression (Jhang et al., 2016).

An inhibition of NLRP3 has also been reported by investigators assessing the anti-inflammatory properties of two other dietary polyphenols, morin (Dhanasekar and Rasool, 2016) and ferulic acid (Doss et al., 2016) in a rat model of acute gout.

Other studies have investigated the beneficial effects of natural compounds in experimental gout models (Martin et al., 2009; Sabina et al., 2011; Huang et al., 2012). Although those studies did not take into full consideration their effect on IL-1 $\beta$ pathway, the overall result was an inhibition of the crystal-induced inflammatory process.

Some interesting findings were produced using butyrate, a short-chain fatty acid produced in the colon by the fermentation of insoluble dietary fibers (Cleophas et al., 2016). This substance has been shown to suppress urate crystalinduced IL-1 $\beta$ production and expression by the inhibition of the histone deacetylase (HDAC) 8, an enzyme that regulates the expression and activity of various proteins. Although the exact mechanism of action is not clear, butyrate did not increase IL-Ra or TGF $\beta 1$, classically involved in the resolution phase of the inflammatory response to pathogenic crystals.

Recently, it has been demonstrated that a high-fiber diet and acetate, a short-chain fatty acid resulting from the metabolism of fiber by gut microbiota, induce faster resolution of the inflammatory response triggered in mice after injection of MSU crystals into the knee joint. In particular, acetate promotes caspase-dependent apoptosis of neutrophils associated with reduced NF-kB activity, lower IL-1 $\beta$ tissue concentations, increased production of anti-inflammatory mediators, such as TGF- $\beta$, IL-10 and annexin A1, and enhanced efferocytosis (Vieira et al., 2017).

\section{REFERENCES}

Akahoshi, T., Namai, R., Murakami, Y., Watanabe, M., Matsui, T., Nishimura, A., et al. (2003). Rapid induction of peroxisome proliferator-activated receptor gamma expression in human monocytes by monosodium urate monohydrate crystals. Arthritis Rheum. 48, 231-239. doi: 10.1002/art.10709

Burt, H. M., Jackson, J. K., and Rowell, J. (1989). Calcium pyrophosphate and monosodium urate crystal interactions with neutrophils: effect of crystal size and lipoprotein binding to crystals. J. Rheumatol. 16, 809-817.

Camell, C., Goldberg, E., and Dixit, V. D. (2015). Regulation of Nlrp3 inflammasome by dietary metabolites. Semin. Immunol. 27, 334-342. doi: 10.1016/j.smim.2015.10.004

Capsoni, F., Ongari, A. M., Reali, E., and Catania, A. (2009). Melanocortin peptides inhibit urate crystal-induced activation of phagocytic cells. Arthritis Res. Ther. 11, R151. doi: 10.1186/ar2827

Chen, Y. H., Hsieh, S. C., Chen, W. Y., Li, K. J., Wu, C. H., Wu, P. C., et al. (2011). Spontaneous resolution of acute gouty arthritis is associated with rapid induction of the anti-inflammatory factors TGF $\beta 1$, IL-10 and soluble TNF

\section{CONCLUSION}

Several mechanisms seem to be involved in resolving crystalinduced inflammation. Although some are linked to exogenous dietary factors, an acute attack is limited by molecules that are activated or expressed following the attack itself through a finely tuned self-regulating mechanism.

As outlined above, these molecules can act at different molecular levels, by affecting cell-crystal interactions, gene expression and cytokine trapping. As regard to IL- $1 \beta$ production process, Figure 2 evidences the substances involved upstream at the mRNA and inflammasome inhibition level, and downstream at IL- $1 \beta$ production, IL- $1 \beta$ release and IL-1R1 signaling level.

Although the precise mechanism of action of some of these factors has not been fully characterized, the final outcome is a diminished IL- $1 \beta$ production.

Considering the importance of IL-1 blocking agents in reducing acute attacks, firstly noted with regard to IL-1Ra anakinra, and then to the anti-IL-1 $\beta$ monoclonal antibody canakinumab, every molecule capable to reduce IL- $1 \beta$ production could represent a potential therapeutic target and have a positive impact on the clinical practice.

\section{AUTHOR CONTRIBUTIONS}

All authors listed, have made substantial, direct and intellectual contribution to the work, and approved it for publication.

\section{FUNDING}

This work was supported by Institutional funds (DOR1658992/16) from the University of Padova.

\section{ACKNOWLEDGMENT}

The authors are grateful to Linda Inverso for the linguistic revision of this manuscript.

receptors and the intracellular cytokine negative regulators CIS and SOCS3. Ann. Rheum. Dis. 70, 1655-1663. doi: 10.1136/ard.2010.145821

Cleophas, M. C., Cri san, T. O., Lemmers, H., Toenhake-Dijkstra, H., Fossati, G., Jansen, T. L., et al. (2016). Suppression of monosodium urate crystal-induced cytokine production by butyrate is mediated by the inhibition of class I histone deacetylases. Ann. Rheum. Dis. 75, 593-600. doi: 10.1136/annrheumdis-2014206258

Cumpelik, A., Ankli, B., Zecher, D., and Schifferli, J. A. (2016). Neutrophil microvesicles resolve gout by inhibiting C5a-mediated priming of the inflammasome. Ann. Rheum. Dis. 75, 1236-1245. doi: 10.1136/annrheumdis2015-207338

Dayer, J. M. (2002). Evidence for the biological modulation of IL-1 activity: the role of IL-1Ra. Clin. Exp. Rheumatol. 20, S14-S20.

Dhanasekar, C., and Rasool, M. (2016). Morin, a dietary bioflavonol suppresses monosodium urate crystal-induced inflammation in an animal model of acute gouty arthritis with reference to NLRP3 inflammasome, hypo-xanthine phospho-ribosyl transferase, and inflammatory mediators. Eur. J. Pharmacol. 786, 116-127. doi: 10.1016/j.ejphar.2016.06.005 
Dinarello, C. A. (2014). An expanding role for interleukin-1 blockade from gout to cancer. Mol. Med. 20, S43-S58. doi: 10.2119/molmed.2014. 00232

Doss, H. M., Dey, C., Sudandiradoss, C., and Rasool, M. K. (2016). Targeting inflammatory mediators with ferulic acid, a dietary polyphenol, for the suppression of monosodium urate crystal-induced inflammation in rats. Life Sci. 148, 201-210. doi: 10.1016/j.lfs.2016.02.004

Dubois, C. M., Ruscetti, F. W., Palaszynski, E. W., Falk, L. A., Oppenheim, J. J., and Keller, J. R. (1990). Transforming growth factor beta is a potent inhibitor of interleukin 1 (IL-1) receptor expression: proposed mechanism of inhibition of IL-1 action. J. Exp. Med. 172, 737-744. doi: 10.1084/jem.172. 3.737

Getting, S. J., Allcock, G. H., Flower, R., and Perretti, M. (2001). Natural and synthetic agonists of the melanocortin receptor type 3 possess antiinflammatory properties. J. Leukoc. Biol. 69, 98-104.

Getting, S. J., Lam, C. W., Chen, A. S., Grieco, P., and Perretti, M. (2006). Melanocortin 3 receptors control crystal-induced inflammation. FASEB. J. 20, 2234-2241. doi: 10.1096/fj.06-6339com

Huang, J., Zhu, M., Tao, Y., Wang, S., Chen, J., Sun, W., et al. (2012). Therapeutic properties of quercetin on monosodium urate crystal-induced inflammation in rat. J. Pharm. Pharmacol. 64, 1119-1127. doi: 10.1111/j.2042-7158.2012. 01504.x

Jhang, J. J., Lu, C. C., and Yen, G. C. (2016). Epigallocatechin gallate inhibits urate crystals-induced peritoneal inflammation in C57BL/6 mice. Mol. Nutr. Food Res. 60, 2297-2303. doi: 10.1002/mnfr.201600106

Joosten, L. A., Crisan, T. O., Azam, T., Cleophas, M. C., Koenders, M. I., van de Veerdonk, F. L., et al. (2016). Alpha-1-anti-trypsinFc fusion protein ameliorates gouty arthritis by reducing release and extracellular processing of IL-1 $\beta$ and by the induction of endogenous IL1Ra. Ann. Rheum. Dis. 75, 1219-1227. doi: 10.1136/annrheumdis-2014206966

Joosten, L. A., Netea, M. G., Fantuzzi, G., Koenders, M. I., Helsen, M. M., Sparrer, H., et al. (2009). Inflammatory arthritis in caspase 1 gene-deficient mice: contribution of proteinase 3 to caspase 1-independent production of bioactive interleukin-1beta. Arthritis Rheum. 60, 3651-3662. doi: 10.1002/art. 25006

Joosten, L. A., Netea, M. G., Mylona, E., Koenders, M. I., Malireddi, R. K., Oosting, M., et al. (2010). Engagement of fatty acids with toll-like receptor 2 drives interleukin- $1 \beta$ production via the ASC/caspase 1 pathway in monosodium urate monohydrate crystal-induced gouty arthritis. Arthritis Rheum. 62, 3237-3248. doi: 10.1002/art.27667

Kumagai, Y., Watanabe, W., Kobayashi, A., Sato, K., Onuma, S., and Sakamoto, H. (2001). Inhibitory effect of low density lipoprotein on the inflammationinducing activity of calcium pyrophosphate dihydrate crystals. J. Rheumatol. 28, 2674-2680.

Lioté, F., Prudhommeaux, F., Schiltz, C., Champy, R., Herbelin, A., OrtizBravo, E., et al. (1996). Inhibition and prevention of monosodium urate monohydrate crystal-induced acute inflammation in vivo by transforming growth factor betal. Arthritis Rheum. 39, 1192-1198. doi: 10.1002/art.178 0390718

Liu-Bryan, R., and Lioté, F. (2005). Monosodium urate and calcium pyrophosphate dihydrate (CPPD) crystals, inflammation, and cellular signaling. Joint Bone Spine 72, 295-302. doi: 10.1016/j.jbspin.2004.12.010

Martin, W. J., Herst, P. M., Chia, E. W., and Harper, J. L. (2009). Sesquiterpene dialdehydes inhibit MSU crystal-induced superoxide production by infiltrating neutrophils in an in vivo model of gouty inflammation. Free. Radic. Biol. Med. 47, 616-621. doi: 10.1016/j.freeradbiomed.2009. 05.035

Martinon, F., Pétrilli, V., Mayor, A., Tardivel, A., and Tschopp, J. (2006). Goutassociated uric acid crystals activate the NALP3 inflammasome. Nature 440, 237-241. doi: 10.1038/nature04516

Murakami, Y., Akahoshi, T., Kawai, S., Inoue, M., and Kitasato, H. (2002). Antiinflammatory effect of retrovirally transfected interleukin-10 on monosodium urate monohydrate crystal-induced acute inflammation in murine air pouches. Arthritis Rheum. 46, 2504-2513. doi: 10.1002/art. 10468

Netea, M. G., van de Veerdonk, F. L., van der Meer, J. W., Dinarello, C. A., and Joosten, L. A. (2015). Inflammasome-independent regulation of IL-1-family cytokines. Annu. Rev. Immunol. 33, 49-77. doi: 10.1146/annurev-immunol032414-112306

Oliviero, F., Sfriso, P., Scanu, A., Fiocco, U., Spinella, P., and Punzi, L. (2013). Epigallocatechin-3-gallate reduces inflammation induced by calcium pyrophosphate crystals in vitro. Front. Pharmacol. 4:51. doi: 10.3389/fphar. 2013.00051

Ortiz-Bravo, E., Sieck, M. S., and Schumacher, H. R. Jr. (1993). Changes in the proteins coating monosodium urate crystals during active and subsiding inflammation. Immunogold studies of synovial fluid from patients with gout and of fluid obtained using the rat subcutaneous air pouch model. Arthritis Rheum. 36, 1274-1285. doi: 10.1002/art.1780360912

Punzi, L., Scanu, A., Ramonda, R., and Oliviero, F. (2012). Gout as autoinflammatory disease: new mechanisms for more appropriated treatment targets. Autoimmun. Rev. 12, 66-71. doi: 10.1016/j.autrev.2012. 07.024

Rose, D. M., Sydlaske, A. D., Agha-Babakhani, A., Johnson, K., and Terkeltaub, R. (2006). Transglutaminase 2 limits murine peritoneal acute gout-like inflammation by regulating macrophage clearance of apoptotic neutrophils. Arthritis Rheum. 54, 3363-3371. doi: 10.1002/art.22137

Sabina, E. P., Nagar, S., and Rasool, M. (2011). A role of piperine on monosodium urate crystal-induced inflammation-an experimental model of gouty arthritis. Inflammation 34, 184-192. doi: 10.1007/s10753-010-9222-3

Scanu, A., Luisetto, R., Oliviero, F., Gruaz, L., Sfriso, P., Burger, D., et al. (2015). High-density lipoproteins inhibit urate crystal-induced inflammation in mice. Ann. Rheum. Dis. 74, 587-594. doi: 10.1136/annrheumdis-2013203803

Scanu, A., Oliviero, F., Gruaz, L., Galozzi, P., Luisetto, R., Ramonda, R., et al. (2016). Synovial fluid proteins are required for the induction of interleukin-1 $\beta$ production by monosodium urate crystals. Scand. J. Rheumatol. 45, 384-393. doi: 10.3109/03009742.2015.1124452

Scanu, A., Oliviero, F., Gruaz, L., Sfriso, P., Pozzuoli, A., Frezzato, F., et al. (2010). High-density lipoproteins downregulate CCL2 production in human fibroblastlike synoviocytes stimulated by urate crystals. Arthritis Res. Ther. 12, R23. doi: 10.1186/ar2930

Scanu, A., Oliviero, F., Ramonda, R., Frallonardo, P., Dayer, J. M., and Punzi, L. (2012). Cytokine levels in human synovial fluid during the different stages of acute gout: role of transforming growth factor $\beta 1$ in the resolution phase. Ann. Rheum. Dis. 71, 621-624. doi: 10.1136/annrheumdis-2011200711

Schauer, C., Janko, C., Munoz, L. E., Zhao, Y., Kienhöfer, D., Frey, B., et al. (2014). Aggregated neutrophil extracellular traps limit inflammation by degrading cytokines and chemokines. Nat. Med. 20, 511-517. doi: 10.1038/nm.3547

Steiger, S., and Harper, J. L. (2013). Neutrophil cannibalism triggers transforming growth factor $\beta 1$ production and self regulation of neutrophil inflammatory function in monosodium urate monohydrate crystal-induced inflammation in mice. Arthritis Rheum. 65, 815-823. doi: 10.1002/art.37822

Terkeltaub, R. A., Dyer, C. A., Martin, J., and Curtiss, L. K. (1991). Apolipoprotein (apo) E inhibits the capacity of monosodium urate crystals to stimulate neutrophils. Characterization of intraarticular apo E and demonstration of apo E binding to urate crystals in vivo. J. Clin. Invest. 87, 20-26. doi: 10.1172/ JCI114971

Terkeltaub, R. A., Smeltzer, D., Curtiss, L. K., and Ginsberg, M. H. (1986). Low density lipoprotein inhibits the physical interaction of phlogistic crystals and inflammatory cells. Arthritis Rheum. 29, 363-370. doi: 10.1002/art.1780290309

Vieira, A. T., Galvão, I., Macia, L. M., Sernaglia, É. M., Vinolo, M. A. R., Garcia, C. C., et al. (2017). Dietary fiber and the short-chain fatty acid acetate promote resolution of neutrophilic inflammation in a model of gout in mice. J. Leukoc. Biol. 101, 275-284. doi: 10.1189/jlb.3A1015-453RRR

Yagnik, D. (2014). Macrophage derived platelet activating factor implicated in the resolution phase of gouty inflammation. Int. J. Inflam. 2014:526496. doi: 10.1155/2014/526496

Yagnik, D. R., Evans, B. J., Florey, O., Mason, J. C., Landis, R. C., and Haskard, D. O. (2004). Macrophage release of transforming growth factor betal during resolution of monosodium urate monohydrate crystal-induced inflammation. Arthritis Rheum. 50, 2273-2280. doi: 10.1002/art.20317

Yen, J. H., Lin, L. C., Chen, M. C., Sarang, Z., Leong, P. Y., Chang, I. C., et al. (2015). The metastatic tumor antigen 1-transglutaminase-2 pathway is involved in selflimitation of monosodium urate crystal-induced inflammation by upregulating TGF- $\beta 1$. Arthritis Res. Ther. 19, 65. doi: 10.1186/s13075-015-0592-7 
Youm, Y. H., Nguyen, K. Y., Grant, R. W., Goldberg, E. L., Bodogai, M., Kim, D., et al. (2015). Ketone body $\beta$-hydroxybutyrate blocks the NLRP3 inflammasomemediated inflammatory disease. Nat. Med. 21, 263-269. doi: 10.1038/nm.3804

Conflict of Interest Statement: The authors declare that the research was conducted in the absence of any commercial or financial relationships that could be construed as a potential conflict of interest.
Copyright (C) 2017 Oliviero and Scanu. This is an open-access article distributed under the terms of the Creative Commons Attribution License (CC BY). The use, distribution or reproduction in other forums is permitted, provided the original author(s) or licensor are credited and that the original publication in this journal is cited, in accordance with accepted academic practice. No use, distribution or reproduction is permitted which does not comply with these terms. 